Honam Mathematical J. 35 (2013), No. 2, pp. 163-171

http://dx.doi.org/10.5831/HMJ.2013.35.2.163

\title{
THE BOREL-CANTELLI LEMMA UNDER PAIRWISE EXTENDED NEGATIVE QUADRANT DEPENDENCE
}

\author{
Mi-Hwa Ko
}

\begin{abstract}
By extending the negatively quadrant dependence, the paper puts forth the concept of extended negative quadrant dependence. A generalization of the second Borel-Cantelli lemma is obtained under extended negative quadrant dependence. Some applications are also introduced.
\end{abstract}

\section{Introduction}

The definitions, properties and applications regarding negative quadrant dependence(NQD) can be found in Lehmman(1966) and Block et al.(1982). We call two events A and B are extended negatively quadrant dependent(ENQD) if there exists a constant $M>0$ such that

$$
P(A \cap B) \leq M P(A) P(B) .
$$

Recall that two events $A$ and $B$ are called negatively quadrant depen$\operatorname{dent}(\mathrm{NQD})$ if (1.1) holds when $M=1$; they are called positively quadrant dependent(PQD) if the inequality (1.1) holds in the reverse direction when $M=1$. Obviously, NQD events must be ENQD events. On the other hand, for some PQD events, it is possible to find a corresponding a positive constant $M$ such that (1.1) holds. Therefore the ENQD events in that it can reflect not only a negative quadrant dependence but also a positive one, to some extent. (See Ko and Tang(2008) and $\operatorname{Liu}(2009)$.)

Received March 5, 2013. Accepted March 19, 2013.

2010 Mathematics Subject Classification. 60F15.

Key words and phrases. Borel-Cantelli lemma, Extended negative quadrant dependence, Generalization of the Borel-Cantelli lemma. 
We call random variables $X$ and $Y$ extended negatively quadrant dependent if there exists a constant $M>0$ such that

$$
\begin{aligned}
P(X \leq x, Y \leq y) & \leq M P(X \leq x) P(Y \leq y) \\
(\text { or } P(X>x, Y>y) & \leq M P(X>x) P(Y>y))
\end{aligned}
$$

holds for all $x, y$.

In the following example, the extended negatively quadrant dependent random variables $X$ and $Y$ can be taken as negatively quadrant dependent or positive quadrant dependent since there are no restrictions on the dependence structure between $X$ and $Y$.

Example. If $X$ is possibly valued at $x_{1} \leq y_{1}$. Then, $X$ and $Y$ are extended negatively quadrant dependent. In fact, for any $x$ and $y$ such that

$$
P(X \leq x) P(Y \leq y)=0,
$$

(1.3) $P(X \leq x, Y \leq y) \leq M P(X \leq x) P(Y \leq y)$ for a constant $M \geq 0$

hold. Additionally, for any $x$ and $y$ such that $P(X \leq x) P(Y \leq y) \neq 0$, take $M=1 / \min \left\{P\left(X=x_{1}\right), P\left(Y=y_{1}\right)\right\}$ then (1.3) still holds.

The following Borel-Cantelli lemma plays an exceptionally important role in probability theory: if $A_{1}, A_{2}, \cdots$ is a sequence of events on common probability space $(\Omega, \mathcal{F}, \mathcal{P})$ and if $\sum_{n=1}^{\infty} P\left(A_{n}\right)<\infty$, then $P\left(\limsup A_{n}\right)=0$; if $A_{1}, A_{2}, \cdots$ is a sequence of independent events and if $\sum_{n=1}^{\infty} P\left(A_{n}\right)=\infty$, then $P\left(\lim \sup A_{n}\right)=1$, where $\lim \sup A_{n}=$ $\cap_{n=1}^{\infty} \cup_{k=n}^{\infty} A_{k}$.

Erdös and Renyi(1959) investigated that the independence condition in the second part of the Borel-Cantelli lemma can be replaced by the weaker condition of pairwise independence of events $A_{1}, A_{2}, \cdots$. They also proved that the condition of pairwise independence of events $A_{1}, A_{2}, \cdots$ can be replaced by the weaker condition $P\left(A_{i} A_{j}\right) \leq P\left(A_{i}\right)$ $P\left(A_{j}\right)$ for every $i$ and $j$ such that $i \neq j$. Recently, $\operatorname{Petrov}(2002,2004)$ and Chandra, T.K.(2008) studied a generalization of the Borel-Cantelli lemma. Especially, note that $\operatorname{Petrov}(2002)$ investigated the following Theorem 2.1 only for the case $M \geq 1$.

In this paper we consider the Borel-Cantelli lemma under extended negative quadrant dependence. As applications we also obtain sufficient condition of strong law of large numbers for extended negatively quadrant dependent random variables. 


\section{Results}

Theorem 2.1. Let $A_{1}, A_{2}, \cdots$ be a sequence of pairwise ENQD events i.e. there exists a constant $M>0$ such that

$$
P\left(A_{i} A_{j}\right) \leq M P\left(A_{i}\right) P\left(A_{j}\right)
$$

for all $i$ and $j(i \neq j)$. If

$$
\sum_{n=1}^{\infty} P\left(A_{n}\right)=\infty
$$

then

$$
\begin{aligned}
& P\left(\limsup A_{n}\right) \geq 1 / M \text { for } M>1, \\
& P\left(\lim \sup A_{n}\right)=1 \text { for } 0<M \leq 1 .
\end{aligned}
$$

Proof. As the proof of Theorem 2.1 in $\operatorname{Petrov}(2002)$ the proof is based on the inequality of Chung and Erdös(1952). For arbitrary events $A_{1}, A_{2}, \cdots$, $A_{n}$, we have

$$
P\left(\cup_{k=1}^{n} A_{k}\right) \geq\left(\sum_{k=1}^{n} P\left(A_{k}\right)\right)^{2} / \sum_{k, j=1}^{n} P\left(A_{k} A_{j}\right) .
$$

Let

$$
S=\sum_{k, j=n, k \neq j}^{N} P\left(A_{k}\right) P\left(A_{j}\right) \text { and } T=\sum_{k=n}^{N} P\left(A_{k}\right) .
$$

It follows from condition (2.1) that

$$
\sum_{k, j=n}^{N} P\left(A_{k} A_{j}\right) \leq M S+T .
$$

Petrov(2002) proved (2.3). For completeness we repeat it here.

If $M>1,(2.7)$ implies the inequality

$$
\sum_{k, j=n}^{N} P\left(A_{k} A_{j}\right) \leq M S+T \leq M(S+T) .
$$

We also have

$$
S=\left(\sum_{k=n}^{N} P\left(A_{k}\right)\right)^{2}-\sum_{k=n}^{N}\left(P\left(A_{k}\right)\right)^{2} .
$$

Therefore, 


$$
\begin{aligned}
S+T & =\left(\sum_{k=n}^{N} P\left(A_{k}\right)\right)^{2}-\sum_{k=n}^{N}\left(P\left(A_{k}\right)\right)^{2}+\sum_{k=n}^{N} P\left(A_{k}\right) \\
& \leq\left(\sum_{k=n}^{N} P\left(A_{k}\right)\right)^{2}+\sum_{k=n}^{N} P\left(A_{k}\right) \\
& =T^{2}+T .
\end{aligned}
$$

By (2.5)-(2.10), we obtain

$$
\begin{aligned}
P\left(\cup_{k=n}^{N} A_{k}\right) & \geq M^{-1} T^{2}\left(T^{2}+T\right)^{-1} \\
& \geq M^{-1}\left(1+T^{-1}\right)^{-1} \\
& =M^{-1}\left\{1+\left(\sum_{k=n}^{N} P\left(A_{k}\right)\right)^{-1}\right\}^{-1} .
\end{aligned}
$$

It follows from condition (2.2) that for a fixed $n$

$$
1+\left(\sum_{k=n}^{N} P\left(A_{k}\right)\right)^{-1} \rightarrow 1 \text { as } N \rightarrow \infty .
$$

Thus,

$$
\liminf _{N \rightarrow \infty} P\left(\cup_{k=n}^{N} A_{k}\right) \geq M^{-1}
$$

and

$$
P\left(\cup_{k=n}^{\infty} A_{k}\right) \geq M^{-1}
$$

Let $B_{n}=\cup_{k=n}^{\infty} A_{k}$, Then, we observe that $B_{1} \supset B_{2} \supset \cdots$ and

$$
\cap_{n=1}^{\infty} B_{n}=\lim \sup A_{n} .
$$

From (2.13) and (2.14) we obtain

$$
\lim P\left(B_{n}\right)=P\left(\cap_{n=1}^{\infty} B_{n}\right)=P\left(\lim \sup A_{n}\right)
$$

and the inequality

$$
P\left(\limsup A_{n}\right) \geq M^{-1}
$$

by $(2.13)-(2.15)$.

If $0<M \leq 1,(2.7)$ implies the inequality

$$
\begin{aligned}
\sum_{k, j=n}^{N} P\left(A_{k} A_{j}\right) & \leq M S+T \\
& \leq S+T .
\end{aligned}
$$


It follows from $(2.5),(2.7),(2.9),(2.10)$ and $(2.16)$ that

$$
\begin{aligned}
P\left(\cup_{k=n}^{N} A_{k}\right) & \geq\left(\sum_{k=n}^{N} P\left(A_{k}\right)\right)^{2} / \sum_{k, j=n}^{N} P\left(A_{k} A_{j}\right) \\
& \geq T^{2}(S+T)^{-1} \\
& \geq T^{2}\left(T^{2}+T\right)^{-1} \\
& \geq\left(1+T^{-1}\right)^{-1} \\
& =\left\{1+\left(\sum_{k=n}^{N} P\left(A_{k}\right)\right)^{-1}\right\}^{-1} .
\end{aligned}
$$

It follows from (2.12) and (2.17) that

$$
P\left(\cup_{k=n}^{\infty} A_{k}\right) \geq 1 .
$$

Hence by (2.13), (2.14), (2.15) and (2.18) we obtain

$$
P\left(\lim \sup A_{n}\right) \geq 1 \text {. }
$$

The proof is complete.

Remark. Recently Petrov $(2002,2004)$ found that (2.3) is satisfied and $P\left(A_{i} A_{k}\right) \leq H P\left(A_{i}\right) P\left(A_{k}\right)$ for all $i, k>N$ such that $i \neq k$ and for some constants only $H \geq 1$ and $N$, then $P\left(\limsup A_{n}\right) \geq H$. He $\operatorname{did}$ not considered for $0<H<1$.

\section{Applications}

Theorem 3.1. Let $\left\{X_{n}, n \geq 1\right\}$ be a sequence of pairwise extended negatively quadrant dependent random variables i.e., there exists a constant $M>0$ such that

$$
P\left(X_{i} \leq x_{i}, X_{j} \leq X_{j}\right) \leq M P\left(X_{i} \leq x_{i}\right) P\left(X_{j} \leq x_{j}\right)
$$

for all $i$ and $j(i \neq j)$ and all $x_{i}, x_{j}$. If

$$
\sum_{n=1} P\left(X_{n} \leq x_{n}\right)=\infty
$$

then

$$
P\left(\lim \sup \left\{X_{n} \leq x_{n}\right\}\right) \geq 1 / M \text { for } M>1,
$$

$$
P\left(\lim \sup \left\{X_{n} \leq x_{n}\right\}\right)=1 \text { for } 0<M \leq 1 .
$$


Proof. Let $A_{k}=\left\{X_{k} \leq x_{k}\right\}$. Then $A_{k}$ 's are pairwise extended negatively quadrant dependent. Hence,by Theorem 2.1 the desired results (3.3) and (3.4) follow.

Theorem 3.2. Let $\left\{X_{n}, n \geq 1\right\}$ be a sequence of pairwise extended negatively quadrant dependent random variables and $\left\{a_{n}, n \geq 1\right\}$ a sequence of positive numbers. If

$$
S_{n} / a_{n} \rightarrow 0 \text { a.s. as } n \rightarrow \infty
$$

and

$$
\sup _{n \geq 1} a_{n-1} / a_{n} \leq M<\infty
$$

for some positive $M$ then

$$
\sum_{n=1}^{\infty} P\left\{\left|X_{n}\right|>a_{n}\right\}<\infty
$$

where $S_{n}=\sum_{i=1}^{n} X_{i}$.

Proof. If $S_{n} / a_{n} \rightarrow 0$ a.s., then $X_{n} / a_{n} \rightarrow 0$ a.s. as $n \rightarrow \infty$. Thus putting

$$
\begin{gathered}
X_{n}^{+}=\max \left\{0, X_{n}\right\}, \\
X_{n}^{-}=\max \left\{0,-X_{n}\right\},
\end{gathered}
$$

we also have

$$
X_{n}^{+} / a_{n} \rightarrow 0 \text { a.s. }
$$

and

$$
X_{n}^{-} / a_{n} \rightarrow 0 \text { a.s. }
$$

Define

$$
A_{n}=\left[X_{n}^{+}>\frac{1}{3} a_{n}\right] \text { and } B_{n}=\left[X_{n}^{-}>\frac{1}{3} a_{n}\right], n \geq 1 .
$$

Note that $A_{i}$ 's and $B_{i}$ 's are pairwise extended negatively quadrant dependent events. Hence, for some positive constant $M>0$ we have

$$
\begin{aligned}
& P\left(A_{k} \cap A_{j}\right) \leq M P\left(A_{k}\right) P\left(A_{j}\right) \text { for } k \neq j, \\
& P\left(B_{k} \cap B_{j}\right) \leq M P\left(B_{k}\right) P\left(B_{j}\right) \text { for } k \neq j .
\end{aligned}
$$

By Theorem 2.1 if $\sum_{n=1}^{\infty} P\left[X_{n}^{+}>\frac{1}{3} a_{n}\right]=\infty$, then $P\left[\limsup A_{k}\right] \geq \frac{1}{M}$ contrary to the almost sure convergence of $X_{n}^{+} / a_{n} \rightarrow 0$. Hence,

$$
\sum_{n=1}^{\infty} P\left[X_{n}^{+}>\frac{1}{3} a_{n}\right]<\infty
$$


By the similar consideration we have

$$
\sum_{n=1}^{\infty} P\left[X_{n}^{-}>\frac{1}{3} a_{n}\right]<\infty
$$

Thus

$$
\begin{aligned}
& \sum_{n=1}^{\infty} P\left[\left|X_{n}\right|>a_{n}\right] \\
= & \sum_{n=1}^{\infty} P\left[X_{n}^{+}+X_{n}^{-}>a_{n}\right] \\
\leq & \sum_{n=1}^{\infty} P\left[X_{n}^{+}>\frac{1}{3} a_{n}\right]+\sum_{n=1}^{\infty} P\left[X_{n}^{-}>\frac{1}{3} a_{n}\right] \\
< & \infty
\end{aligned}
$$

which completes the proof.

Theorem 3.3. Let $\left\{X_{n}, n \geq 1\right\}$ be a sequence of pairwise extended negatively quadrant dependent random variables with the same distribution $F(x)$. If $S_{n} / n \rightarrow 0$ a.s., then $E\left|X_{1}\right|<\infty$.

Proof. In Theorem 3.2 set $a_{n}=n$ and take into account that $X$ 's are equidistributed. Then by Theorem 3.2 we get $\sum_{n=1}^{\infty} P\left[\left|X_{n}\right|>n\right]>\infty$. Thus $E\left|X_{1}\right|<\infty$.

In the appendix we introduced the properties of extended negative quadrant dependence needed to prove main results.

\section{Appendix}

Theorem A.1. Two random variables $X$ and $Y$ satisfy that there exists a positive constant $M>0$ such that

$$
P(X \leq x, Y \leq y) \leq M P(X \leq x) P(Y \leq y),
$$

for all $x$ and $y$.

Then there is a positive constant $M^{\prime}>0$ such that

$$
P(X>x, Y>y) \leq M^{\prime} P(X>x) P(Y>y),
$$

for all $x$ and $y$. 
Proof. When $P(X>x, Y>y)=0$ obviously (A.2) holds. Now assume $P(X>x, Y>y) \neq 0$.

$$
\begin{aligned}
P(X>x, Y>y)= & P(X>x)-P(X>x, Y \leq y) \\
= & P(X>x)-P(Y \leq y)+P(X \leq x, Y \leq y) \\
\leq & P(X>x)+P(Y>y)-1+M P(X \leq x) P(Y \leq y) \\
= & P(X>x)+P(Y>y)-1 \\
& +M(1-P(X>x))(1-P(Y>y)) \\
\leq & (M-1)+M P(X>x) P(Y>y) .
\end{aligned}
$$

If $0<M \leq 1$, then $P(X>x, Y>y) \leq M P(X>x) P(Y>y)$.

If $M>1$, then

$$
\begin{aligned}
& \qquad \begin{aligned}
P(X>x, Y>y) \leq & \left(M+\frac{M-1}{P(X>x) P(Y>y)}\right) P(X>x) P(Y>y)
\end{aligned} \\
& \text { where } M^{\prime}=M+\frac{M-1}{P(X>x) P(Y>y)} \geq M>1 .
\end{aligned}
$$

Theorem A.2. If $(X, Y)$ is extended negatively quadrant dependent, then for nondecreasing functions $f$ and $g(f(X), g(Y))$ is also extended negatively quadrant dependent.

\section{References}

[1] Block, H.W., Savits, T.H. and Shaked, M., Some concepts of negative dependence, Ann. Probab. 10 (1982), 765-772.

[2] Chung, K.L. and Erdös, P., On the application of Borel-Cantelli lemma, Trans. Amer. Soc. 72 (1952), 179-186.

[3] Chandra, T.K., The Borel-Cantelli lemma under dependence conditions, Statist. Probab. Lett. 78 (2008), 390-395.

[4] Erdös, P. and Renyi, A., On Cantor's series with convergent $\sum \frac{1}{q_{n}}$, Ann. Univ. Sei. Vudapest. Sect. Math. 2 (1959), 93-109.

[5] Ko, B. and Tang, Q., Sums of dependent nonnegative random variables with subexponential tails, J. Appl. Prob. 45 (2008), 85-94.

[6] Lehmann, E.L., Some concepts of dependence, Ann. Math. Statist. 37 (1966), 1137-1153.

[7] Liu, L., Precise large deviations for dependent variables with heavy tails, Statist. Probab. Lett. 79 (2009), 1290-1298.

[8] Petrov, V.V., A note on the Borel-Cantelli lemma, Statist. Probab. Lett. 58 (2002), 283-286.

[9] Petrov, V.V., A generalization of the Borel-Cantelli lemma, Statist. Probab. Lett. 67 (2004), 233-239. 


\section{Mi-Hwa Ko}

Division of Mathematics and Informational Statistics,

WonKwang University,

Jeonbuk 570-749, Korea.

E-mail : songhack@wonkwang.ac.kr 